\title{
Face Recognition under Varying Illumination Using Green's Function- based Bidimensional Empirical Mode Decomposition and Gradientfaces
}

\author{
Zhi-Jun Yang, Xue He, Wen-Yi Xiong, Xiang-Fei Nie \\ School of electronics and information engineering, Chongqing Three Gorges University,404000, wanzhou, Chongqing, China.
}

\begin{abstract}
A novel face recognition approach under varying illumination condition based on Green's function in tension-based bid imensional empirical mode decomposition (GiT-BEMD) and gradient faces (GBEMDGF) is present. Firstly, face image was illumination normalization by discrete cosine transform that an appropriate number of DCT coefficients are truncated in logarithm domain. And then, two intrinsic mode functions (IMFs) that relevant of intrinsic physical significances of face images are produced by Gi T-BEMD. At the same time, gradient faces is used to improve the high frequency component of face images and to extract illumination insensitive facial feature. The facial feature of discriminately are fused using IMFs and illumination insensitive feature. Secondly, the principal component analysis is adopted to reduce the dimension of face image. The nearest neighbourhood classifier based on cosine distance is implemented for face classification. Experimental results on Yale B database and CUM PIE face database demonstrate that the present technique is robust to varying lighting resource.
\end{abstract}

\section{Introduction}

It is a well-known fact that variation under illumination condition could seriously affect the performance of face recognition system [ll 1 2]. The changes of same face image under different illumination condition are bigger compared with the change of different face image under same illumination condition. Within the past decade, many researchers were focused on illumination problem and many algorithms that deal with lighting under vary condition were proposed. In general, those algorithms are divided into three categories [3]. The first category is illumination pre-processing and illumination normalization. Histogram equalization (HE) [4], Gamma correction and Logarithm transform [5] are widely used for illumination normalization. However, those algorithms are consideration the global characteristic of face image rather than the local detail feature that is important to face recognition. Thus those algorithms cannot obtain ideal face recognition result. The second category attempts to extract the illumination invariant features. In [6], the author proposes a method to extract illumination insensitive facial feature under varying lighting condition called Gradientfaces in gradient domain. However, the data of face image was not reduced, so the calculation is complication .In [7], the author uses the gradient angle as the input feature. It generates the gradient vectors in different form, and then computes the gradient angle. The third category, which handle the illumination problem by construct the face model. In [8], the author present a method that a set of images in fixed pose, but under different lighting condition, can form light convex cone, which can generate different face images with different light direction. Nevertheless, this method needs a variety of train samples or face images under different lighting resource, which is not an ideal method for real time environment.

In this paper, we present a novel face illumination processing technique based on bidimensional empirical mode decomposition and gradient faces. Discrete cosine transform is adopted to normal face image. The high frequency intrinsic mode functions (IMFs) of face image are produced by GiT-BEMD. Gradient faces is adopted to enhance the high frequency detail of face image and to extract the Gradient faces, which is illumination insensitive facial feature. Two kinds of features are fused to improve the performance of image recognition. The BEMD can achieve intrinsic mode functions, which is physical feature relating to face image. Gradient faces extract illumination invariant feature. Two kinds of feature are fusion to as discriminative facial feature. The PCA is applied to reduce the dimension of face image and the nearest neighbourhood classifier based on cosine distance is utilized for face classification. Experiment results in this paper demonstrate that our method is robust to light change with different direction. The flowchart of this paper is shown in figure1.

The rest of this correspondence is organized as follows. In section 2, logarithm transform, green's 
function-based bidimensional empirical mode decomposition and gradient faces are present in detail. Many experimental results are described in section 3 . Finally, the conclusion is given in section 4.

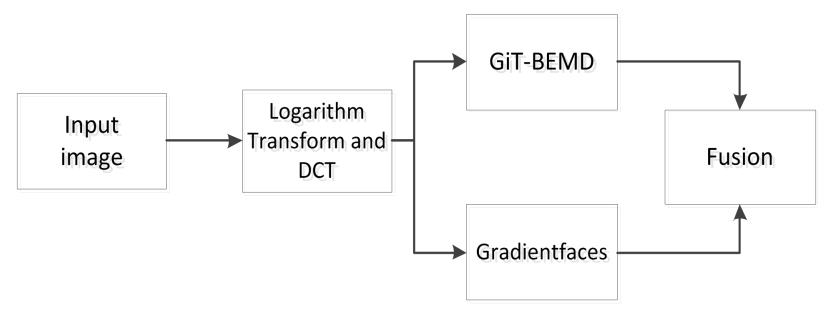

Fig.1 The Flowchart of Our Method

\section{Methodologies}

\subsection{Logarithm transform}

The reflectance model [9] can be expressed as follows:

$$
I(x, y)=R(x, y) \times L(x, y)
$$

Where $I(x, y)$ is arbitrary image pixel value, $R(x, y)$ is the reflectance which is fast change part of the image and $L(x, y)$ is the illuminance which is slow change part of the image. The logarithmic operation is used to eq. (1).

$$
\ln [I(x, y)]=\ln [R(x, y)]+\ln [L(x, y)]
$$

The reflectance component $R(x, y)$ is multiplied by the lighting component $L(x, y)$ in time domain. However, it is adding when the image was transformed into logarithm domain. Thus, the lighting component is subtracted from face images to eliminate the illumination effective in logarithm domain, which is similar to highpass filter..

\subsection{GiT-BEMD}

Bidimensional empirical mode decomposition decomposes face image into a series of intrinsic mode functions (IMFs) which is high frequency and low frequency component of the face image and a residue. In this paper, the GiT-BEMD [10] is present here based on a representation of upper and lower surface envelopes via suitable Green's functions for spline interpolation including surface tension. The difference between canonical BEEMD and GiT-BEMD are the process of estimating the upper and lower surfaces and limiting the number of iterations per BIEMD to a few iterations only. In this method, a data point/pixel is considered as a local maximum (minimum), if its value is strictly higher (lower) than all of its neighbors. Let $P=\left\{p_{i} \mid i=1,2 \cdots N\right\}$ be a set of local minima (maxima) of an $x \times y$-dimensional data matrix such that it exists a small (large) neighbourhood around any such local optimal point $p_{i}$ on which the pixel value is never larger (small) than $f\left(x_{i}, y_{i}\right)$ at $p_{i}$.Local extrema occur only at critical points. Let $D(x, y)=f_{x x} f_{y y}-\left(f_{x y}\right)^{2}$. If $D>0$ at a critical point, then the critical $p_{i}$ is a local extremum. The signs of $f_{x x}$ and $f_{y y}$ determine whether the point is a maximum or a minimum. If $D \leq 0$ at a critical point, then the point $p_{i}$ is a saddle point.

In BEMD, spline interpolation is basically used to find the smoothest surface passing through a grid of irregularly spaced extrema, either maxima or minima. In this word, it is proposed to employ Green's functions, deduced from proper data constraints, to expand the interpolating surface under tension. Interpolation with Green's functions implies that the points of the interpolating envelope surface can be expressed as

$$
s\left(x_{u}\right)=\sum_{n=1}^{N} w_{n} \Phi\left(x_{u}, x_{n}\right)
$$

Where $x_{u}$ denotes any point where the surface is unknown, $x_{n}$ represents the nth data constraint, $\Phi\left(x_{u}, x_{n}\right)$ is the Green's function and $w_{n}$ is the respective weight in the envelope representation.

\subsection{Gradientfaces}

The Gradientfaces [6] can be defined as follows:

For face image $I(x, y)$, the relationship between neighboring point $(x, y)$ and $(x+\Delta x, y)$ defined as follows.

$$
I(x+\Delta x, y)=R(x+\Delta x, y) \times L(x+\Delta x, y)
$$

According to the common assumption that $L$ is approximately smooth and changing slowly:

$$
\begin{aligned}
& I(x+\Delta x, y)-I(x, y) \\
& =R(x+\Delta x, y) \times L(x+\Delta x, y)-R(x, y) L(x, y) \\
& \approx(R(x+\Delta x, y)-R(x, y)) L(x, y)
\end{aligned}
$$

Considering the limitation of the above equality (11), we have:

$$
\frac{\partial I(x, y)}{\partial x} \approx L(x, y) \frac{\partial R(x, y)}{\partial x}
$$

Similarly, we have:

$$
\frac{\partial I(x, y)}{\partial y} \approx L(x, y) \frac{\partial R(x, y)}{\partial y}
$$

Dividing (6) by (7), we have: 


$$
\frac{I_{y}}{I_{x}} \approx \frac{R_{y}}{R_{x}}
$$

Where $I_{x}=\frac{\partial I(x, y)}{\partial x}$ and $I_{y}=\frac{\partial I(x, y)}{\partial y}$ is the gradient of face image under $x$ and $y$ direction, respectively. $R_{x}=\frac{\partial R(x, y)}{\partial x}$ and $R_{y}=\frac{\partial R(x, y)}{\partial y}$ are the gradient of reflectance component under $x$ and $y$ direction, respectively.

Considering the common assumption that $R$ can be considered as an illumination insensitive component, so as to the radio of $I_{y}$ and $I_{x}$.

Therefore the gradient faces is defined as follows:

$$
G=\arctan \left(\frac{I_{y}}{I_{x}}\right), G \in[-\pi, \pi]
$$

In order to compute gradient faces, we need to calculate the gradient of face image in $x$ and $y$ direction. We are smoothing face images by convolving Gaussian kernel function firstly. The Gaussian kernel function is expressed as following:

$$
G(x, y, \sigma)=\frac{1}{2 \pi \sigma^{2}} \exp \left(-\frac{x^{2}+y^{2}}{2 \sigma^{2}}\right)
$$

Where $\sigma$ is standard deviation.

\subsection{The fusion of facial feature}

In this paper, the Green's Function - based Bidimensional Empirical Mode Decomposition is wielded to generate two IMFs and the illumination insensitive feature is extracted. Two kinds of features are combined to come up with distinguished facial feature. The algorithmic view of the proposed method is summarized as follows:

Input: Original face image $I$.

Output: The GBEMDGF of $I^{\prime}$.

1. Logarithmic transform using eq. (2).

2. Apply Discrete Cosine Transform to discard low-frequency DCT coefficient on $I$ to gain normalization face image $f$.

3. Apply GiT-BEMD on $f$ to achieve IMF $g$. $g(i, j)=g_{1}(i, j)+g_{2}(i, j) ; i=1,2 \cdots M ; j=1,2 \cdots N$. Where $M$ and $N$ are the size of face image.

4. Apply Gradientfaces on $f$ to extract illumination insensitive feature $q(i, j)$ using eq. (9).

5. Feature fusion by $I^{\prime}=\left[\begin{array}{l}g(i, j) \\ q(i, j)\end{array}\right]$ to attain lighting insensitive facial feature.

\section{Experiments}

\subsection{Face Database}

Our experiments are conducted on the Yale $\mathrm{B}$ face database and CUM PIE face database. The Yale B face database contains images of ten individuals with nine pose and 64 illuminations per pose. The frontal face images of all subjects, each with 64 different illuminations are used to evaluate the algorithm. In our experiment, all face images are cropped and resized to $128 \times 128$ and divided into five subsets based on the angle of lighting direction. Subset 1 (0o-12o), subset 2 (13o-25o), subset 3 (26o-50o), subset 4 (51o-77o) and subset 5 (above 78o) has 70,120,120,140,190 images, respectively. CUM PIE database contains 68 subjects with 41368 face images in total. We use illumination subset (C27) to as the experiment samples. In our experiment, we use 68 subjects with 1428 face images, each with 21 different illuminations for testing. All images are cropped and resized to $68 \times 68$.

\subsection{Experiment results on Yale B}

Fig.2 shows five images for one individual from different subsets and corresponding face images processed with our method.

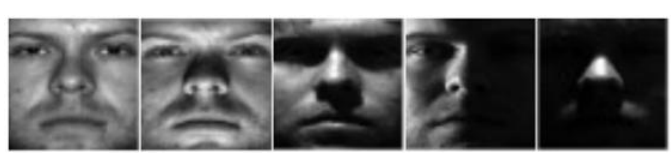

(a)

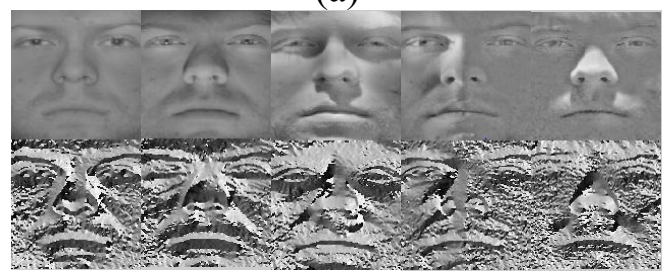

(b)

Figure2 (a) Original Face Images and (B) Face Image Processed with Our Method.

In order to compare with the results from important literature, the first experiment is devised to subset one as reference subset and other images from subset 2 to subset 5 as query subsets. Comparison results are shown in Table I. Bidimensional empirical mode decomposition (BEMD) and our method has the same experiment condition.

Table I Performance Comparison on Yale B Database

\begin{tabular}{|l|l|l|l|l|l|}
\hline \multirow{2}{*}{ Method } & \multicolumn{5}{|l|}{ Face Recognition(\%) } \\
\cline { 2 - 6 } & $\begin{array}{l}\text { Subset } \\
2\end{array}$ & Subset3 & Subset4 & Subset5 & $\begin{array}{l}\text { Averag } \\
\text { e }\end{array}$ \\
\hline BEMD & 98.33 & 98.33 & 89.29 & 43.68 & 77.89 \\
\hline
\end{tabular}




\begin{tabular}{|l|l|l|l|l|l|}
\hline GF[6] & 100 & 100 & 98.75 & 98.95 & 99.3 \\
\hline QI[11] & 99.3 & 61.9 & 34.1 & 23.3 & 54.65 \\
\hline LBP[12] & 100 & 97.6 & 65.2 & 44.4 & 76.8 \\
\hline Our method & 100 & 100 & 100 & 100 & 100 \\
\hline
\end{tabular}

The second experiment is devised to use every subset on Yale B database as train subset and other subset from subset 1 to subset 5 expect for train subset as test subset to indicate the effectiveness of our method. When subset 1 as train set, the average recognition of subset 2, subset 3 , subset 4 and subset 5 are $100 \%$. When subset 2 as train set, the average recognition of subset 1 , subset 3 , subset 4 and subset 5 are $100 \%$. When subset 3 as train set, the average recognition of subset 1 , subset 2 , subset 4 and subset 5 are $100 \%$. When subset 4 as train set, the average recognition of subset 1 , subset 2 , subset 3 and subset 5 are $100 \%$. When subset 5 as train set, the average recognition of subset 1 , subset 2 , subset 3 and subset 4 are $100 \%$.

\subsection{Experiment results on CUM PIE}

Some face image from CUM PIE database are shown in Fig.3.

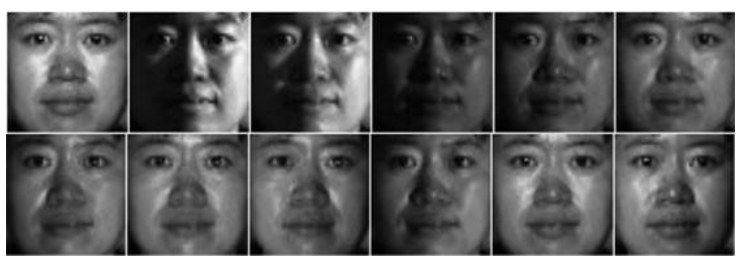

(c)

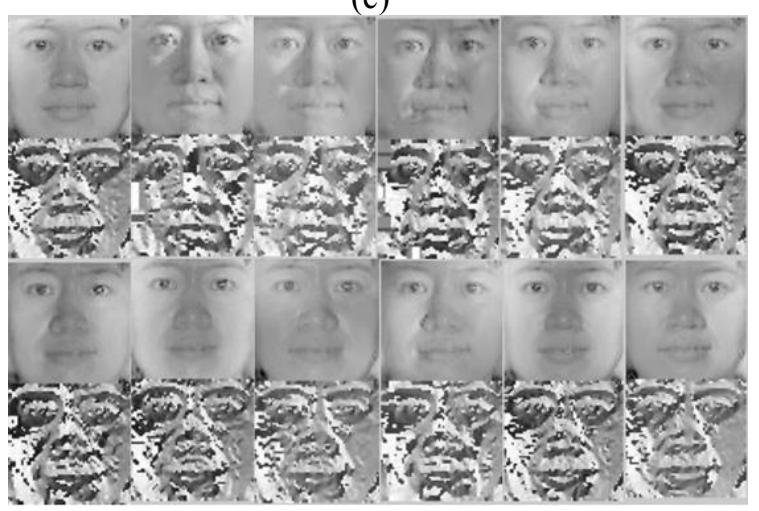

(d)

Figure3 (c) Original Images and (D) Face Images Processed With Our Method

We conduct one experiment on this face database. In this experiment, we chose one person in every subject as train image and others as test samples. Thus we have 68 reference images and 1360 query images. The experiment results are shown in Table II. BEMD, GF and our method have same experiment condition. The experiment results of SQI and MSR are from [6]. The recognition rate of BEMD, GF, SQI, MSR and our method are $90.22 \%$, $95.07 \%, 77.94 \%, 62.07 \%$ and $100 \%$. Thus our method can achieve ideal face recognition rate compared with BEMD, GF, SQI and MSR.

Table II Experiment Result Comparison with Different Methods on Cum Pie Database

\begin{tabular}{|l|l|l|l|l|l|}
\hline & \multicolumn{4}{|l|}{ Methods } \\
\cline { 2 - 6 } & BEMD & GF & SQI[6] & MSR[6] & $\begin{array}{l}\text { Our } \\
\text { Method } \\
\text { s }\end{array}$ \\
\hline $\begin{array}{l}\text { Rate } \\
\text { \%) }\end{array}$ & 90.22 & $\begin{array}{l}95 . \\
07\end{array}$ & 77.94 & 62.07 & 100 \\
\hline
\end{tabular}

\section{Conclusions}

In this correspondence, we propose a method based on the bidimensional empirical mode decomposition. And the gradient faces algorithm to process face image under varying lighting. The two IMFs of face image was decomposed in logarithm domain. And then, the gradient faces was adopted to enhance the high frequency component of face images after reconstruction. The PCA is used to extract facial feature and the nearest neighborhood classifier based on cosine distance is used to classify. Experimental results on Yale B database and CUM PIE database demonstrate that the method in our paper is robust algorithm for face recognition under varying illumination. We will test the effectiveness of this technique in bigger face database in the future.

\section{Acknowledgment}

This research was financially supported by the Project Foundation of Chongqing Municipal Education Committee (KJ121114).

\section{References}

1. Han H, Shan S, Chen X, et al. A comparative study on illumination preprocessing in face recognition [J]. Pattern Recognition, 2013, 46(6):1691-1699.

2. Ochoa-Villegas M A, Nolazco-Flores J A, BarronCano O, et al. Addressing the illumination challenge in two-dimensional face recognition: a survey [J]. Iet Computer Vision, 2015, 9(6):978-992.

3. Baradarani A, Wu Q M J, Ahmadi M. An efficient illumination invariant face recognition framework via illumination enhancement and DD-DTCCWT filtering[J]. Pattern Recognition,2013,46(1) :57-72.

4. Shan S, Gao B, et al. Illumination normalization for robust face recognition against varying lighting conditions[C]//IEEE International Workshop on Analysis and Modelling of Faces and Gestures. IEEE Computer Society, 2003:157-157.

5. Savvides M, Kumar B V K V.Illumination normalization using logarithm transforms for face authentication $[\mathrm{C}] / /$ International Conference on Audio-and Video-Based Biometric Person Authentication. Springer-Verlag,2003:549-556. 
6. T Zhang, Y Y Tang, B Fang . et al, Face recognition under varying illumination using gradientfaces, IEEE Transactions on image processing, 2009, 18(11): 2599-2606.

7. An G, Wu J, Ruan Q. Improved Gradientface used in face recognition under varying illumination $[\mathrm{C}] / /$ IEEE, International Conference on Signal Processing. IEEE, 2010:670-673.

8. A. S. Georghiades, P. N. Belhumeur, and D. J. Kriegman. From few to many: Illumination cone models for face recognition under variable lighting and pose. IEEE Trans. Pattern Anal. Mach. Intell, 23(6):643-660, 2001.

9. Shahamat H, Pouyan A A. Face recognition under large illumination variations using homomorphic filtering in spatial domain[J]. Journal of Visual Communication \& Image Representation, 2014, 25(5):970-977.

10. Al-Baddai S, Al-Subari K, Tomé A M, et al. A green's function-based Bi-dimensional empirical mode decomposition[J]. Information Sciences, 2016, 348:305-321.

11. Shashua A, Riklin-Raviv T. The Quotient Image: Class-Based Re-Rendering and Recognition with Varying Illuminations [J]. Pattern Analysis \& Machine Intelligence IEEE Transactions on, 2001, 23(2):129-139.

12. Ahonen T, Hadid A, Pietikainen M. Face Description with Local Binary Patterns: Application to Face Recognition[J]. IEEE Transactions on Pattern Analysis \& Machine Intelligence, 2006, 28(12):2037-2041. 